\title{
ON THE DESIGN OF INTELLIGENT PUBLIC INFORMATION FACILITIES - TAKE THE PUBLIC SERVICE ROBOT AS AN EXAMPLE
}

\author{
Huiping Dai \\ Shanghai Sanda University, Room 504, No. 12, Lane 792, Fahuazhen Road, Changning District, \\ Shanghai, China 200052
}

\begin{abstract}
In the construction of the smart city, intelligent public facilities are indispensable. As a type of intelligent public facilities, public service robot has some problems in the current application process. For example, technical problems, communication difficulties, lack of guidance and other aspects of the problem, and in view of these problems, in this paper, put forward the corresponding design strategy, hoping to optimize the overall design of the public service robot, improve its comprehensive level, so as to become a booster to improve the public service level of the smart city.
\end{abstract}

\section{KEYWORDS}

Interactive Design, Intelligence, Public Information Facility, Public Service Robot

\section{INTRODUCTION}

In the city, public facilities such as signboards and information inquiry machines can be seen everywhere. These facilities bring a lot of convenience to people's life. With the progress and development of science and technology, the intelligent level of the facilities will gradually improve, bringing people's life with efficiency and convenience that never existed before.

\section{INTELLIGENT PUBLIC INFORMATION FACILITY AND PUBLIC SERVICE ROBOT}

Public information facility is a kind of urban public facilities. Its main function is to provide people with direction guidance, instruction, information, public affairs, etc. With the rapid development of modern science and technology, many changes have taken place in the public information facility. Therefore, a variety of intelligent information facilities have emerged, such as electronic rolling screens, interactive touch screens, public service robots, etc. And a public service robot is a new form of intelligent public information facility, which integrates core technologies such as visual recognition, speech recognition, intelligent interaction, network technology, real-time system, sensor detection, control technology, and system integration. It will become a representative form of intelligent public facilities in the future.

\section{FEATURES OF PUBLIC SERVICE ROBOT}

A public service robot is a kind of intelligent system terminal, which is shaped like a human, has greeting etiquette and navigation services, can speak, sing, interact with people in natural language, has simple actions and facial expressions, can provide public information, guide navigation, realize temperature detection, fire alarm, air quality detection, and other functions. Its main features are as follows: First, a public service robot is a combination of public information facilities and artificial intelligence. The robot combines a variety of 
intelligent interaction technologies. Through intelligent sensing, information processing, and other technologies, the robot can collect various behavior data of people, and then upload it to the intelligent management system. Second, as an intelligent system terminal, after accessing the Internet of things, it can also control the public facilities (such as lighting, access control, etc.) in an area. Third, public service robot integrates various interaction modes. With the continuous development of intelligent interaction technology, people can experience more natural and rich interaction.

\section{THE CURRENT SITUATION OF PUBLIC SERVICE ROBOT}

Nowadays, public service robots can be seen in many public places, such as banks, hospitals, libraries, and other public places. For the interaction experience with the public service robot, the author has conducted random interviews. Some people said that "voice input has problems and can't be accurately recognized," some people said "don't know how to use it, it's better to ask the staff directly," some said that "you can chat a few sentences, but it's basically not very meaningful," and there are many problems. In summary, the main problems are as follows:

\subsection{Problems of Intelligent Identification Technology}

Although speech recognition, face recognition, and other technologies are developing rapidly, as far as robots are concerned, speech recognition and other technologies cannot reach the level of natural communication. People often use voice to communicate with the public service robot. When there are errors in speech recognition, it will lead to communication obstacles. Finally, they need to turn to the real waiter. Therefore, at present, the intelligent identification technology of public service robots is not mature and stable, and it cannot recognize the surrounding world as people do, which directly affects the effectiveness of public service robot interaction design.

\subsection{Matching the Environment}

A public service robot provides services for people in public places. The usual place is basically the entrance or hall of the place, such as the outpatient Hall of the hospital, the railway station hall, etc., while the public place is characterized by open space and dense personnel, and the environment is generally noisy, so it is questionable whether the public service robot based on voice interaction is suitable for use in such an environment. Under the current technology, public service robot does not have very mature speech recognition function, which increases the difficulty of recognition in a noisy environment. In addition, the complexity of people in public places, people from different places, have different dialects, voice, and intonation, which makes recognition more difficult.

\subsection{Problems with User Interaction}

In the beginning, people were curious about the public service robot. However, after the initial contact, many people did not use it again, which shows that the public service robot is not perfect in terms of humanization. Although the public service robot has the functions of singing, chatting and telling jokes, its expression mode is still very stylized, which brings a kind of rigid and cold feeling to people, while the live receptionist has rich emotional expression and more temperature, which is the key difference between the public service robot and the real person.

\subsection{Lack of Effective Operation Guidance}

In the face of new things, people will be curious and confused. Even though new devices are easier to use and more efficient than old ones, people still have psychological barriers to new devices before they learn how to use them. Although the public service robot is considered to be able to talk freely with people, due to the 
current technical limitations, the interaction between robots and people cannot be as flexible as that between people. After a simple attempt to talk, people find that the public service robot does not achieve the desired interaction effect, so they are reluctant to try again, which leads to the public service The robot is idle after a period of time.

\section{THE DESIGN STRATEGY OF INTELLIGENT PUBLIC INFORMATION FACILITY}

\subsection{Improve Technical Level}

Human robot interaction (HRI) involves many disciplines. It has gradually developed into an independent research field. Just how to make robots understand the real world like people is a challenging topic. Therefore, the natural interaction between robot and human will not be easy to achieve, but it can be predicted that with the continuous development of technology, researchers will be able to solve all the key problems, improve the effectiveness of human-robot interaction, so that robot can communicate with people normally, and become an indispensable partner in people's life.

\subsection{Interactive Design Matching with the Environment}

In the public environment, the interaction between public service robots and human is affected by the environment to some extent, so the product design should consider matching with the environment. On the one hand, because the public environment is generally noisy, the voice interaction between public service robots and users is not good. In this regard, other interaction methods can be adopted, such as increasing the size of the screen, mainly touch-screen interaction, supplemented by voice interaction, or at the same time of voice interaction, the screen displays the corresponding visual form information such as subtitles, so that the subtitles can be seen even if the voice cannot be heard clearly. On the other hand, the appearance of the robot, as a part of the interface, should conform to the environmental style. For example, the public service robot in the bank lobby needs to highlight the characteristics of simplicity, lightness, and atmosphere. The public service in the hospital clinic lobby should reflect its fresh, clean and comfortable characteristics.

\subsection{Enhance Emotional Experience Design}

An important element of human-robot interaction is Emotionalization. Public service robot brings good emotional experience to people, which will make them warmer. For example, when receiving children, we can design some interesting games to play with them, so as to arouse a happy atmosphere. In a hospital, when people have a long waiting time, the public service robot will come forward to interact with people, and its attitude should be warm and full of care, so as to alleviate the anxiety of waiting, and also help patients to prepare for treatment. When encountering the elderly, the public service robot can go forward and ask carefully and provide rest, drinking water, walking assistance, and other physical help.

\subsection{Add Operation Instruction}

When a new product appears, users need to learn how to use it, so they need to provide necessary operation instruction information, such as what functions a robot has, what public information it can provide to people, how people get the information they need, and what specific operation steps are. For different users, their cognitive level and knowledge reserve are different, so the way of guidance can also be changed accordingly. Young and middle-aged people are familiar with the operation of electronic equipment. They also know some principles of robots in different degrees, so they can use words, pictures, or short videos to convey the operation instructions to them. But for the older people, because they are not familiar with electronic products, and even some will reject them psychologically, so they can let the professional staff guide them by hand, and they need to pay some patience. In this way, users can better use the public service robot, and this kind of intelligent public information facility can play its role to the maximum extent. 


\subsection{Give Full Play to the Advantages of Multi-Sensory Interaction and Serve the Vulnerable Groups}

In addition to people with physical and mental health, there are also vulnerable groups such as the elderly and the disabled. Therefore, public facilities should not only ensure the normal use of people but also facilitate the use of vulnerable groups, so as to become barrier-free public facilities serving the whole society. As for the public service robot loaded with various intelligent technologies, it can be more friendly to vulnerable groups. It can provide personalized communication methods for different people's perception and operation capabilities. For example, the blind people take touch and hearing as the main sensing channels, and the robot will interact with them in voice or Braille and other ways accordingly. It is worth noting that in addition to providing physiological help to vulnerable groups and reducing barriers in communication, we should also pay attention to psychological care for vulnerable groups, improve the sense of pleasure in the use process, so that they can feel calm and comfortable in the process, and get equal and harmonious communication experience with society, environment, and other people.

\section{CONCLUSION}

A public service robot is a new type of public information facility, which will also be one of the trends of intelligent facilities in the future. However, the current technology level cannot fully meet the needs of people, and there are some defects in the overall design. Public service robot cannot completely replace people, and cannot complete all the work of public service personnel. With the continuous efforts to improve the technical level and strengthen the human-robot interaction design, the development of public service robot will change with each passing day, and gradually become a booster to improve the public service level of the smart city.

\section{REFERENCES}

Weiss. Astrid. et al, 2014. Designing a service robot for public space: an "action and experiences" - Approach. ACM/IEEE International Conference on Human-robot Interaction, pp. 318-319.

Zhang Mang-mang, 2016. Research of Barrier-free Design under the Background of Building Smarter Cities. Disability Research, vol. 1, pp. 39-44. 